\title{
Effect and mechanisms of celastrol on the apoptosis of HOS osteosarcoma cells
}

\author{
YANYANG CHEN, YUNSHENG OU, YONG TAO, HUZHE LIU, HANG YIN, \\ SHENXI ZHONG, HAOYANG YU, ZENGHUI ZHAO and BIN HE
}

\begin{abstract}
Department of Orthopedics, The First Affiliated Hospital of Chongqing Medical University, Yuzhong, Chongqing 400016, P.R. China
\end{abstract}

Received January 20, 2018; Accepted July 19, 2018

DOI: $10.3892 / o r .2018 .6619$

\begin{abstract}
Osteosarcoma is the most common primary malignant tumor of the bone found predominantly in children and teenagers and results in early metastasis and poor prognosis. The present study primarily focused on the impact of celastrol on apoptosis and autophagy of osteosarcoma HOS cells, as well as the related mechanisms. Following the appropriate treatment, the human osteosarcoma cell line HOS was assessed for viability, $\mathrm{Ca}^{2+}$ in cells, apoptosis and changes in cell morphology using Cell Counting Kit-8, flow cytometry, inverted phase contrast microscope, Hoechst staining and transmission electron microscopy. The expression levels of various proteins, including endoplasmic reticulum stress (ERS)-related proteins (Bip, PERK, p-PERK, IRE1 $\alpha$, calnexin, PDI and Erol-L $\alpha$ ), apoptosis-related proteins (CHOP, cleaved caspase-12), mitochondrial apoptosis-related proteins (Bax, Bcl-2 and cytochrome $c$ ), cleaved caspase-3, and autophagy-related proteins (LC3-I, LC3-II and P62) and $\beta$-actin, were assessed with western blotting. Celastrol significantly inhibited the viability of HOS cells in a dose-dependent manner and promoted the expression of ERS-related, apoptosis-related and mitochondrial apoptosis-related proteins. The ERS inhibitor tauroursodeoxycholate promoted celastrol-induced autophagy and apoptosis of HOS cells. Pretreatment with the PERK inhibitor GSK2656157 significantly promoted celastrol-induced death and attenuated HOS cell autophagy. Our results indicated that the ERS pathway and the mitochondrial pathway were involved in celastrol-induced apoptosis of HOS cells. The ERS/PERK pathway may protect HOS cells from apoptosis by celastrol and may play a complicated role in the process of autophagy.
\end{abstract}

Correspondence to: Professor Yunsheng Ou, Department of Orthopedics, The First Affiliated Hospital of Chongqing Medical University, 1 Youyi Road, Yuzhong, Chongqing 400016, P.R. China E-mail: ouyunsheng2001@163.com

Key words: celastrol, osteosarcoma, apoptosis, autophagy, ERS, PERK

\section{Introduction}

Osteosarcoma is the most common primary malignant tumor of the bone found predominantly in children and teenagers and results in early metastasis and poor prognosis. With the development of neoadjuvant chemotherapy and surgical improvement, the five-year survival rate of patients with osteosarcoma is close to $60 \%$ (1). However, adverse drug reactions and drug resistance of osteosarcomas are becoming more and more common, and novel drugs are urgently needed to treat osteosarcoma (2).

Celastrol, a quinone methide triterpenoid, is isolated from the root of Tripterygium wilfordii and is primarily used for the treatment of autoimmune diseases and neurodegenerative diseases $(3,4)$. In recent years, celastrol has exhibited great potential in inducing apoptosis of tumor cells and has shown significant progress for osteosarcoma treatment (5-10). Apoptosis, known as type I-programmed cell death, mainly involves changes in cell morphology (e.g., chromosome condensation or fragmentation), shrinkage or invagination of the cell membrane and apoptotic bodies (11-15). Apoptosis is one principal approach to induce tumor death. The mitochondrial, death receptor and endoplasmic reticulum stress (ERS) pathways are widely accepted to mediate cell apoptosis.

The endoplasmic reticulum (ER) is a very complex organelle and functions in protein synthesis, transmembrane transport, post-translational modification, glycosylation modification, synthesis of phospholipids and cholesterol and control of intracellular calcium homeostasis. When ER homeostasis is disturbed, many unfolded proteins bind to Glucose-regulated protein 78 (Grp78/Bip) and activate the pathways of PERK, activating transcription factor 6 (ATF6) and inositol-requiring enzyme 1 (IRE1) to promote the recovery of ER function and protect cells. However, extended durations or high strength of ERS will induce cell apoptosis mainly through three apoptotic pathways, including CHOP, JNK and caspase-12 $(13,16)$.

The aim of the present study was primarily to explore the effect of celastrol on the HOS human osteosarcoma cell line and the related mechanisms (especially ERS, the mitochondrial pathway and autophagy). We further explored the effect of PERK signaling on ERS and its relationship with apoptosis and autophagy. 


\section{Materials and methods}

Chemicals and cell culture. Celastrol, sodium tauroursodeoxycholate (TUDCA), thapsigargin (TG) and Hoechst 33258 were purchased from Sigma-Aldrich; Merck KGaA (Darmstadt, Germany). Celastrol had a purity higher than $98 \%$. A stock solution $(50 \mathrm{mmol} / \mathrm{l})$ was made in dimethyl sulfoxide (DMSO) (Nanjing KeyGen Biotech, Co.,Ltd.,Nanjing, China) and stored in the dark at $-80^{\circ} \mathrm{C}$. GSK 2656157 was purchased from Selleck Chemicals (Houston, TX, USA). Dulbecco's modified Eagle's medium (DMEM), fetal bovine serum (FBS) and trypsin were all purchased from Gibco ${ }^{\mathrm{TM}}$ (Thermo Fisher Scientific, Inc., Waltham, MA, USA). Cell viability and cytotoxicity test kits [Cell Counting Kit-8 (CCK-8)] and FLUOU-3/AM were obtained from Dojindo Molecular Technologies (Kumamoto, Japan). The Annexin V-propidium iodide (PI) double-staining test kit was purchased from Nanjing KeyGen Biotech. Bip, Calnexin, endoplasmic oxidoreductin-1-like protein $\alpha$ (Ero1-L $\alpha$ ), protein disulfide isomerase (PDI), IRE1 $\alpha$, PERK, CHOP, cleaved caspase-3, cytochrome $c$, Bax, Bcl-2, LC-3, p62 and $\beta$-actin were purchased from Cell Signaling Technology, Inc., (Danvers, MA, USA). Cleaved caspase-12 was purchased from Proteintech Group Inc., (Wuhan, China) and p-PERK was supplied by Santa Cruz Biotechnology (Santa Cruz, CA, USA). HOS cells were purchased from the American Type Culture Collection (ATCC; Manassas, VA, USA) and cultured in DMEM containing $100 \mu \mathrm{g} / \mathrm{ml}$ penicillin, $100 \mu \mathrm{g} / \mathrm{ml}$ streptomycin, and $10 \% \mathrm{FBS}$ (complete medium) at $37^{\circ} \mathrm{C}$ in $5 \% \mathrm{CO}_{2}$.

Cytotoxicity assay. The anti-proliferative effect of celastrol on HOS cells was assessed by after $12 \mathrm{~h}$, they were treated with various concentrations of celastrol $(0,1.5,2.5,3.5$ and $4.5 \mu \mathrm{M})$ for $24 \mathrm{~h}$. Subsequently, $10 \mu \mathrm{l} \mathrm{CCK}-8$ was added to each well, and the plates were incubated in an incubator for 1 to $2 \mathrm{~h}$ at $37^{\circ} \mathrm{C}$ following the manufacturer's instructions. A microplate reader was used to detect the absorbance at $450 \mathrm{~nm}$. Data represented the mean of five replicates. The cell viability was calculated using the following equation: Cell viability $(\%)=$ Average $\mathrm{OD}$ in study group/average OD in control group x $100 \%$, where OD was the optical density. Each assay was performed in triplicate. Based on the results of the cell viability assay, we selected an appropriate celastrol concentration for subsequent experiments.

Examination of the cell ultrastructure by transmission electron microscopy. HOS cells were seeded in 24-well plates at a density of $5 \times 10^{4}$ cells/well, and treated with the celastrol concentration of $3.00 \mu \mathrm{M}$ for $24 \mathrm{~h}$. Subsequently, the culture medium was discarded, and the cells were washed three times with phosphate-buffered saline (PBS) (Nanjing KeyGen Biotech), then fixed with $1 \%$ osmic acid and $2.5 \%$ glutaraldehyde, dehydrated using acetone and gradient ethanol, embedded, solidified, sliced using an ultramicrotome and stained with 3\% uranium acetate-lead citrate. The cells were observed to evaluate morphological changes and images were captured with transmission electron microscopy.

Assessment of apoptosis by Hoechst nuclear staining. HOS cells were seeded in 24-well plates at a density of $5 \times 10^{4}$ cells/well, and treated with the celastrol concentration of $3.00 \mu \mathrm{M}$ for
$24 \mathrm{~h}$. Subsequenlty, the culture medium was discarded, and the cells were washed three times with PBS. After the HOS cells were fixed with $4 \%$ paraformaldehyde for $5 \mathrm{~min}, 10 \mu \mathrm{g} / \mathrm{ml}$ Hoechst $33258(200 \mu \mathrm{l})$ was added to the cells at $37^{\circ} \mathrm{C}$ for $5 \mathrm{~min}$ in the dark. The cells were observed to evaluate apoptotic changes and images were captured with a fluorescence microscope after being washed three times with PBS.

Apoptosis determined by flow cytometry (FCM). HOS cells were seeded in 6-well plates at a density of $5 \times 10^{6}$ cells/well. Suspended and adherent cells were collected after treatment and analyzed with an Annexin V/PI apoptosis kit using FCM.

Measurement of calcium ions. HOS cells were seeded in 6-well plates at a density of $5 \times 10^{6}$ cells/well. Suspended and adherent cells were collected following treatment and analyzed with a FLUO-3/AM $(2 \mu \mathrm{M})$ kit using FCM.

Expression of p-PERK determined by immunofluorescence staining. HOS cells were seeded in 24-well plates at a density of $5 \times 10^{4}$ cells/well, and treated with the celastrol concentration of $3.00 \mu \mathrm{M}$ for $24 \mathrm{~h}$. Subsequently, the cells were washed with PBS, fixed in $4 \%$ freshly prepared formaldehyde for $5 \mathrm{~min}$, and permeabilized in PBS with $0.1 \%$ Triton X-100 for $5 \mathrm{~min}$ at room temperature. Cells were then incubated for $1 \mathrm{~h}$ in blocking solution ( $3 \%$ bovine serum albumin $/ 0.08 \%$ glycine in PBS) and treated with a rabbit polyclonal antibody against p-PERK (1:1,000; cat. no. Sc-32577; Santa Cruz Biotechnology, Santa Cruz, CA, USA) for $2 \mathrm{~h}$ at room temperature. The secondary antibody, fluorescein (FITC)-conjugated AffiniPure goat anti-rabbit (1:200; cat. no. SA00003-2; ProteinTech Group, Inc., Chicago, IL, USA), was applied for $1 \mathrm{~h}$ at room temperature, and nuclei were counterstained with DAPI (Beyotime Institute of Biotechnology, Shanghai, China). Slides were viewed under a fluorescence microscope (Axio Observer A1; Carl Zeiss AG, Oberkochen, Germany).

Western blot analysis. Western blotting was performed to observe the protein expression level. Briefly, at various time-points, cells in the control and celastrol groups were collected and lysed using radioimmunoprecipitation assay (RIPA) buffer (Keygen Biotech, Jiangsu, China) containing protease inhibitor cocktail (Selleck Chemicals, Houston, TX, USA) for $30 \mathrm{~min}$ on ice. The following steps were performed as depicted in our previous study (11).

Statistical analysis. The quantitative data are represented as the mean \pm standard deviation (SD) and comparisons of significant differences were calculated by one-way analysis of variance (ANOVA) with Dunnett's test or unpaired Student's $\mathrm{t}$-test. All statistical analyses were performed using SPSS software (version 22.0; IBM Corp., Armonk, NY, USA). Statistical significance tests were two-tailed, and $\mathrm{P}<0.05$ was considered to indicate a statistically significant difference.

\section{Results}

Celastrol decreases HOS cell viability. The effect of celastrol on cell viability was investigated by exposing HOS cells to various concentrations of celastrol for $24 \mathrm{~h}$ (Fig. 1). The CCK-8 


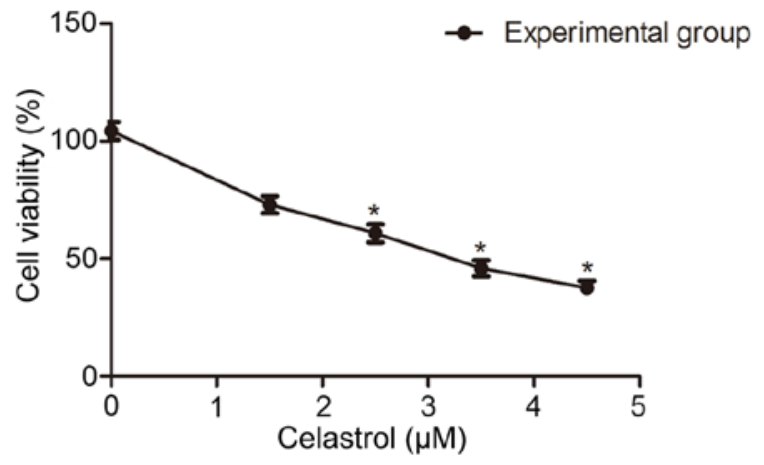

Figure 1. Inhibitory effects of different concentrations of celastrol on the viability of HOS cells for $24 \mathrm{~h}$ were detected by CCK- 8 . "P<0.05 vs. the control group. Cel, celastrol. CCK-8, Cell Counting Kit-8.

assay demonstrated that celastrol could inhibit the viability of HOS cells in a dose-dependent manner and revealed that the $50 \%$ inhibitory concentration $\left(\mathrm{IC}_{50}\right)$ was $3.36 \mu \mathrm{M}$ for HOS cells. Therefore, based on previous experiments, we chose a celastrol concentration of $3.00 \mu \mathrm{M}$ as the treatment condition for the following experiments (10).

Celastrol induces apoptosis of HOS cells. Under inverted phase contrast microscopy, retracted, round and floating cells were observed, and karyopyknosis, condensation, karyorrhexis and other typical apoptotic bodies were observed with Hoechst 33255 nuclear staining following treatment with celastrol for $24 \mathrm{~h}$ (Fig. 2A and B). However, there were no significant changes in the control group. FCM revealed that the apoptosis rates of the 6,12 and 24-h groups were all higher than that of the control group $(\mathrm{P}<0.05)$ (Fig. 2C and D). These results indicated that celastrol induced apoptosis of HOS cells.

The ERS pathway and mitochondrial pathway are involved in apoptosisinduced by celastrol in HOS cells. Under Transmission electron microscopy (TEM), the HOS cells displayed swelling of the ER following celastrol treatment for $24 \mathrm{~h}$, whereas the control group exhibited no changes in the ER (Fig. 3A). FCM demonstrated that the level of calcium in the celastrol (24 h) group was significantly higher than that in the control group (Fig. 3B). In addition, western blotting revealed that at 6 , 12 and $24 \mathrm{~h}$ following treatment using celastrol, the expression levels of ERS-related proteins (Bip, p-PERK, IRE1 $\alpha$, Calnexin, Ero1-L $\alpha$ and PDI), ERS-related apoptosis proteins (CHOP, cleaved caspase-12) and cleaved caspase-3 in HOS cells were all increased, while PERK was decreased compared to those of the control group (Fig. $3 \mathrm{C}$ and D, Fig. $4 \mathrm{~A}$ and $\mathrm{B} ; \mathrm{P}<0.05$ ). These results indicated that ERS was induced by celastrol in HOS cells, and ERS-induced apoptosis was also involved in HOS cell apoptosis induced by celastrol. To exclude false-positive results of the experiment and the influence of DMSO, following treatment with TG, we observed that the expression levels of Bip, CHOP and cleaved caspase-12 in HOS cells all increased, while following treatment with DMSO, all of them decreased, indicating that TG could induce ERS-induced apoptosis but DMSO could not (Fig. 4C and D; $\mathrm{P}<0.05$ ).

Furthermore, western blotting also revealed that following treatment with celastrol for 6,12 and $24 \mathrm{~h}$, Bax and cytochrome $c$ in the cytoplasm, indexes of mitochondrial apoptosis, were increased, while Bcl-2 was decreased (Fig. 5A and B; $\mathrm{P}<0.05$ ). These findings indicated that the mitochondrial pathway was involved in celastrol-induced apoptosis in HOS cells.
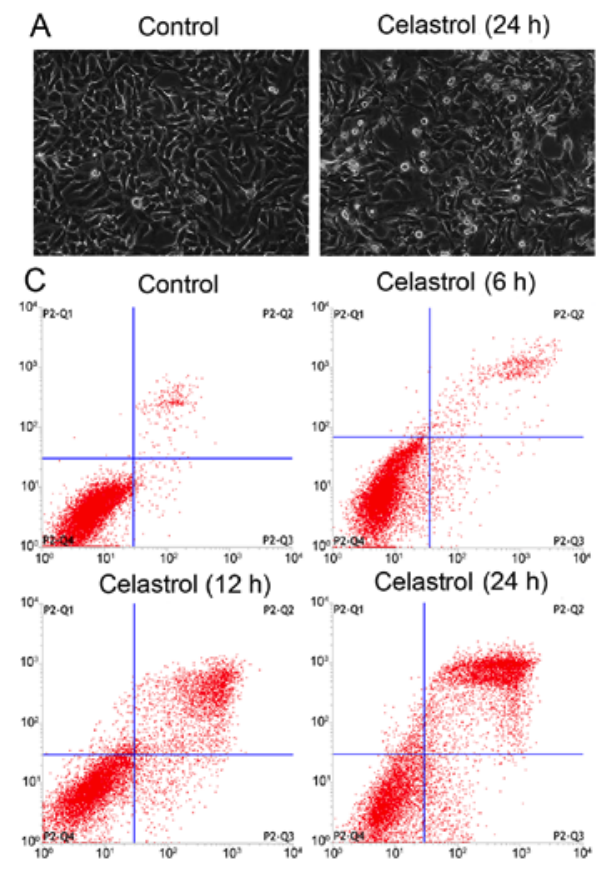

Celastrol $(6 \mathrm{~h})$
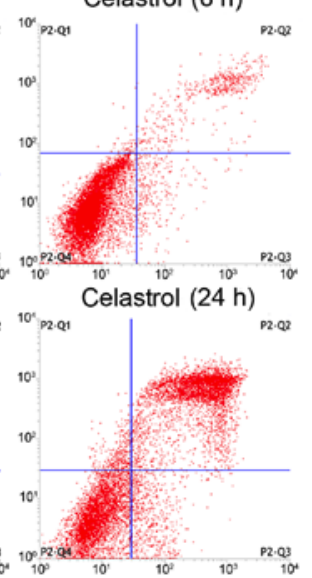

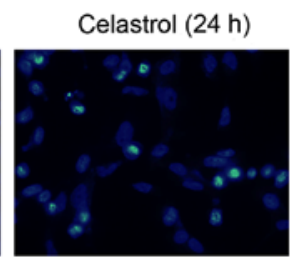

D

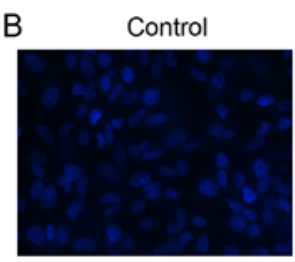

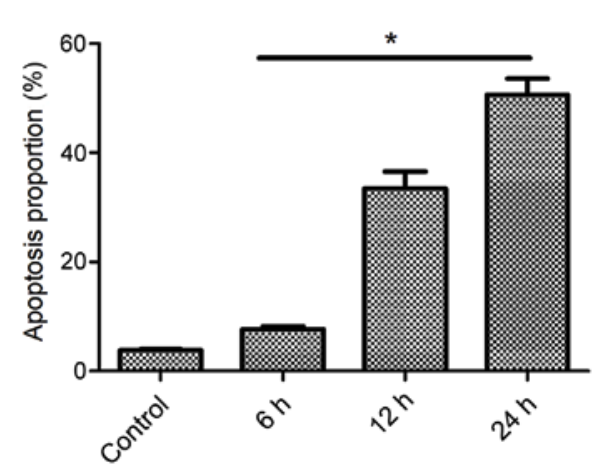

Figure 2. Celastrol induces apoptosis of HOS cells. (A) Following treatment with celastrol for 24 h, the morphology was observed under an inverted microscope (magnification, x200); (B) The apoptotic morphological changes were observed under fluorescence microscopy (magnification, $\mathrm{x} 200$ ). (C) The apoptotic rates at different time-points $(0,6,12$ and $24 \mathrm{~h})$ were analyzed with FCM. (D) The FCM data from three independent experiments are displayed in bar format. ${ }^{*} \mathrm{P}<0.05$ vs. the control group. FCM, flow cytometry. 
A
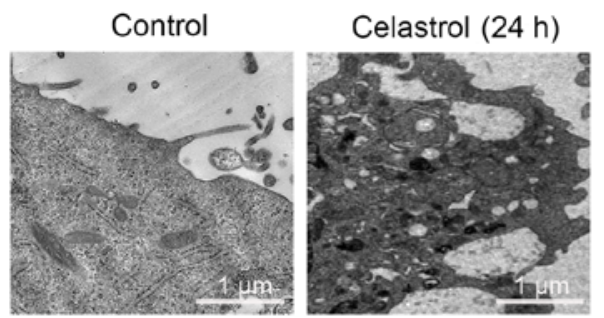

C

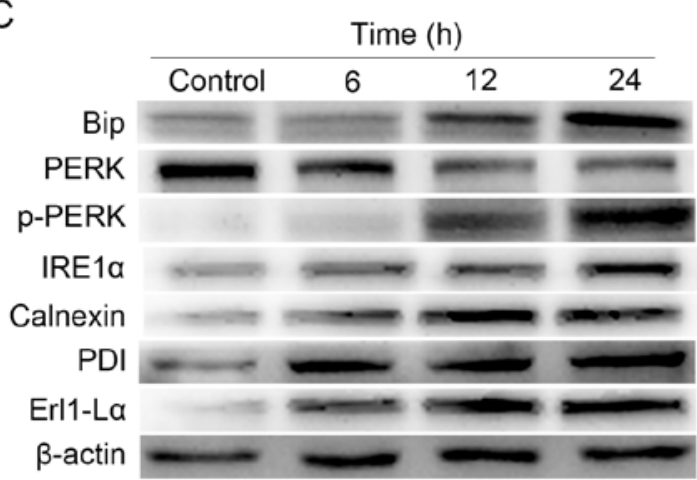

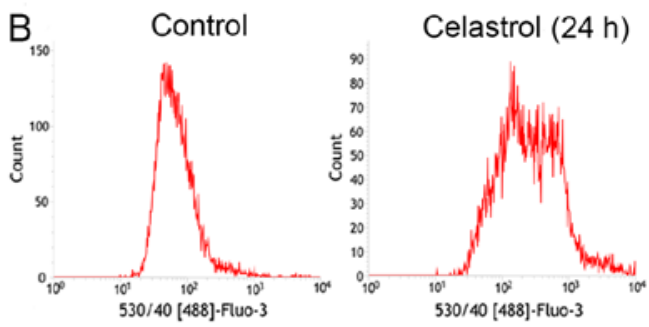

D $\quad$ Bip $ळ$ PERK 口

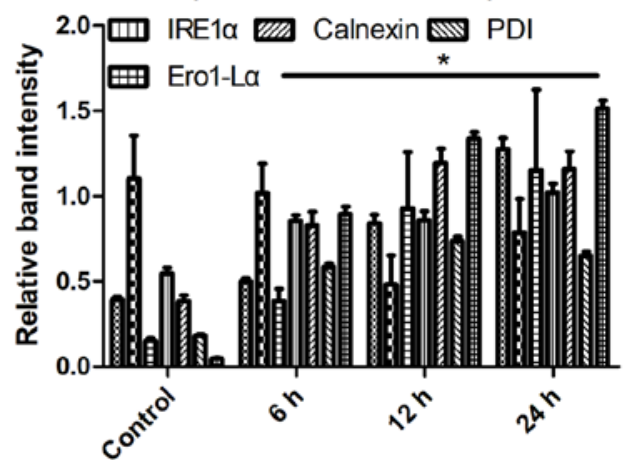

Figure 3. ERS is induced by celastrol. (A) For $24 \mathrm{~h}$ following treatment, the ER was observed by TEM. (B) The level of calcium in cells was determined by FCM. P $<0.05$ vs. the control group. (C and D) At 6,12 and $24 \mathrm{~h}$, whole-cell lysates were prepared to assay Bip, p-PERK, IRE1 $\alpha$, calnexin, Ero1-L $\alpha$, PDI and PERK proteins by western blotting. Data are presented as the mean \pm SD from three independent experiments. ${ }^{P}<0.05$ vs. the control group. ERS, endoplasmic reticulum stress; ER, endoplasmic reticulum; TEM, transmission electron microscopy; FCM, flow cytometry; SD, standard deviation.

A

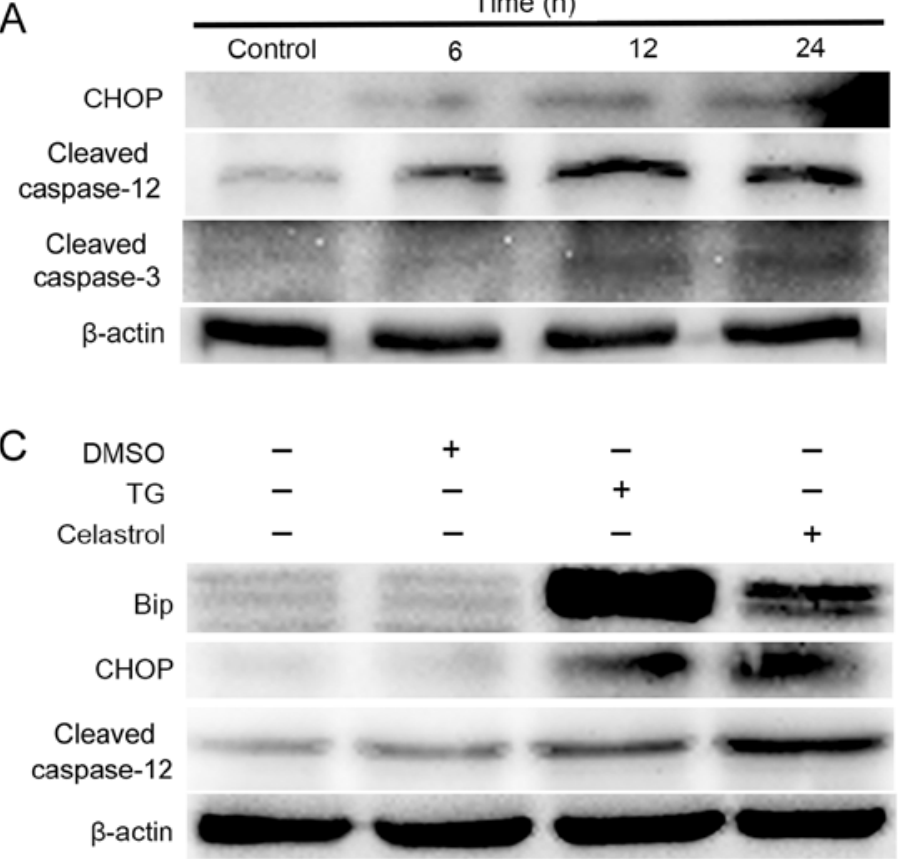

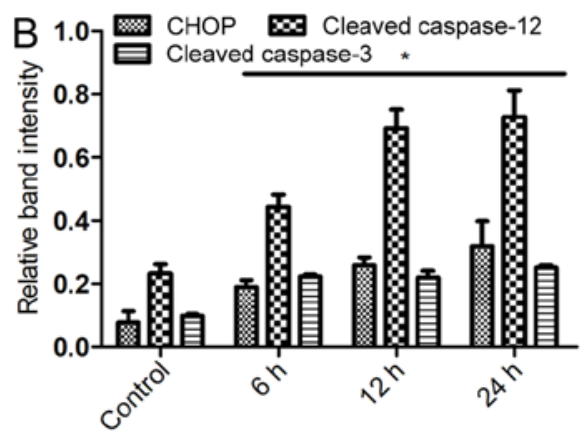

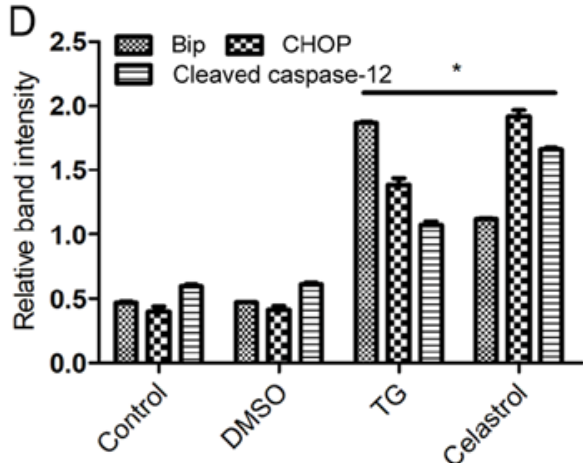

Figure 4. The ERS pathway is involved in celastrol-induced apoptosis in HOS cells. (A and B) At $24 \mathrm{~h}$ following treatment, whole-cell lysates were prepared to assay CHOP, cleaved caspase- 12 and cleaved caspase-3 proteins by western blotting. Data are presented as the mean \pm SD from three independent experiments. ${ }^{*} \mathrm{P}<0.05$ vs. the control group. (C and D) TG was used as a positive control, and $24 \mathrm{~h}$ following treatment, whole-cell lysates were prepared to assay Bip, CHOP and cleaved caspase- 12 proteins by western blotting. Data are presented as the mean $\pm \mathrm{SD}$ from three independent experiments. ${ }^{*} \mathrm{P}<0.05$ vs. the control group. ERS, endoplasmic reticulum stress; SD, standard deviation; TG, thapsigargin.

Apoptosis and autophagy are promoted, while ERS induced by celastrol is suppressed. TUDCA $(500 \mu \mathrm{M})$, an inhibitor of ERS, was used to pretreat HOS cells for $1 \mathrm{~h}$ before celastrol treatment $(17,18)$. FCM demonstrated that pretreatment with TUDCA significantly increased the apoptosis rate compared to celastrol treatment alone (Fig. 6A and B; $\mathrm{P}<0.05$ ). Furthermore, western blotting revealed that pretreatment with TUDCA promoted the expression of Lc-3 II and cleaved caspase-3 compared with 
A

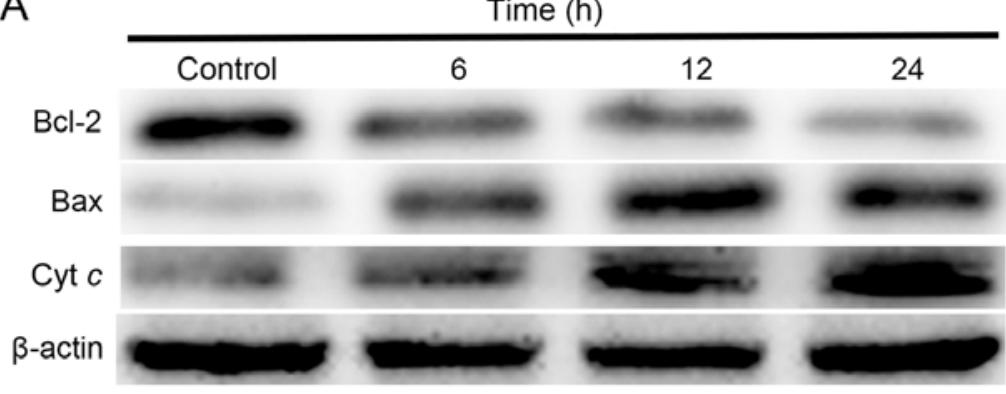

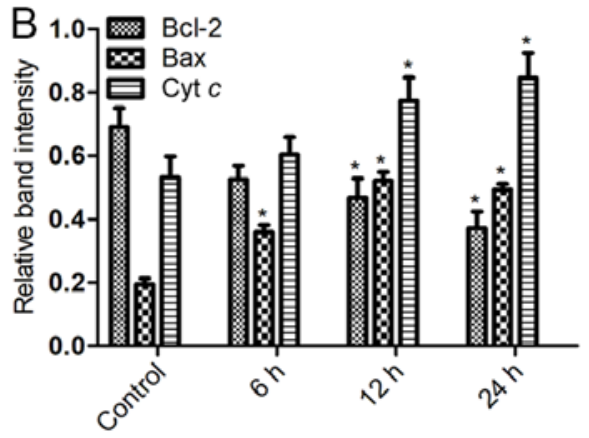

Figure 5. The mitochondrial pathway is involved in celastrol-induced apoptosis in HOS cells. (A and B) At $24 \mathrm{~h}$ after treatment, whole-cell lysate were prepared to assay cytochrome $c$, Bax and Bcl-2 proteins by western blotting, and cytosol fractions were used to assay cytochrome $c$. Data are presented as the mean \pm SD from three independent experiments. ${ }^{*} \mathrm{P}<0.05$ vs. the control group. $\mathrm{SD}$, standard deviation.
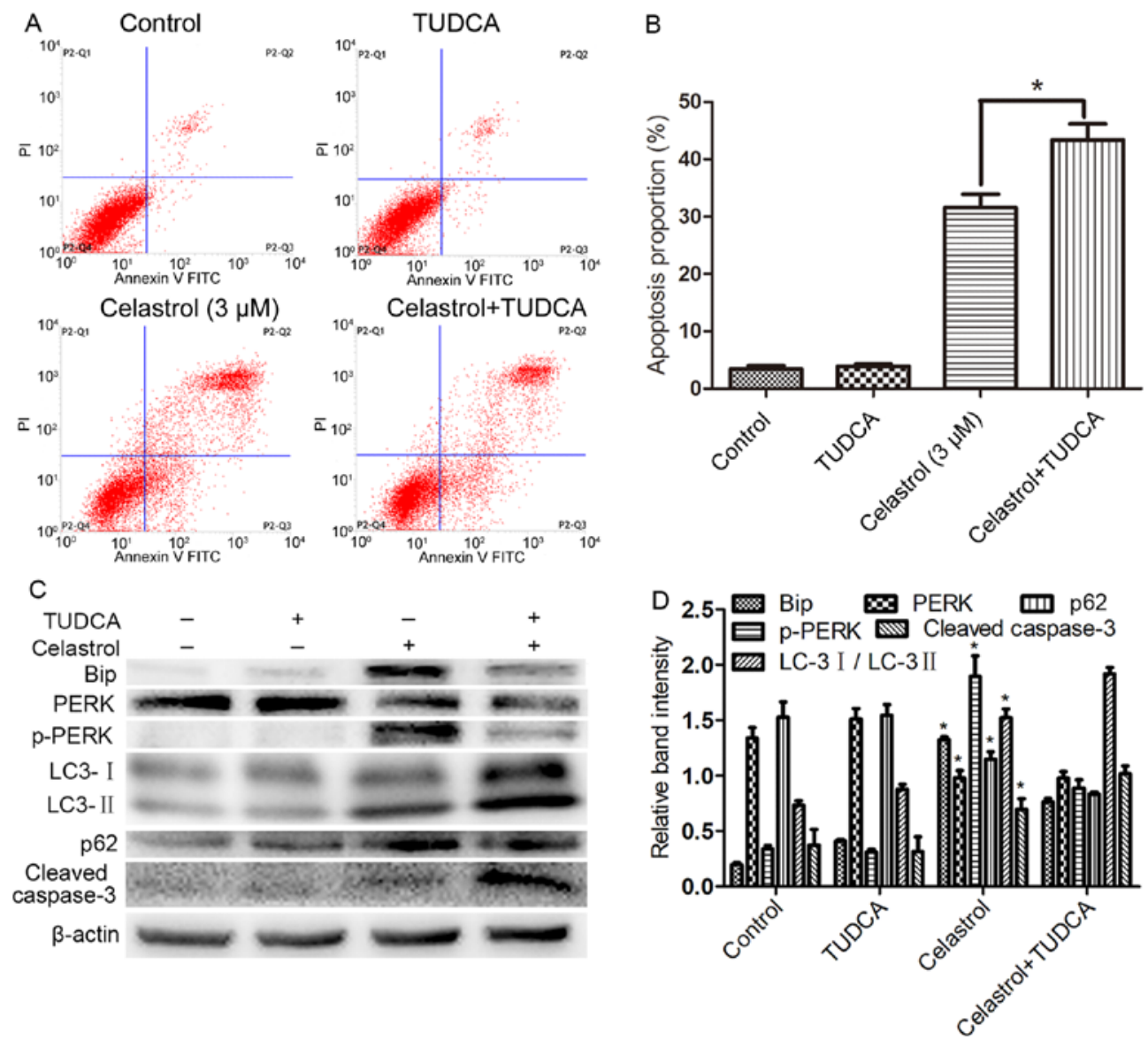

Figure 6. The ERS pathway protects HOS cells from apoptosis. (A and B) At $24 \mathrm{~h}$, following pretreatment with TUDCA, the apoptosis rates of HOS cells were determined by FCM. "P $<0.05$ vs. the celastrol group. (C and D) At $24 \mathrm{~h}$, following pretreatment with TUDCA, whole-cell lysates were prepared to assay Bip, PERK, p-PERK, LC-3, p62, and cleaved caspase-3. Data are presented as the mean \pm SD from three independent experiments. "P<0.05 vs. the control group. ERS, endoplasmic reticulum stress; TUDCA, tauroursodeoxycholate; FCM, flow cytometry; SD, standard deviation.

celastrol alone treatment, while the expression of Bip, p-PERK, and p62 was decreased (Fig. $6 \mathrm{C}$ and $\mathrm{D} ; \mathrm{P}<0.05$ ). All the results indicated that attenuating the ERS of HOS cells would promote the autophagy and apoptosis induced by celastrol.

Apoptosis is promoted, while the PERK signaling pathway is suppressed. The unfolded protein response (UPR) protects HOS cells from apoptosis induced by celastrol. To maintain homeostasis of HOS cells, a large number of unfolded proteins activate the PERK signaling pathway by binding to Bip, and
PERK, which is an important transducer, activates downstream molecular partners and protein secretion related to molecular gene transcription. To explore whether attenuating the expression of PERK signaling pathway members would reverse the protective effect of ERS induced by celastrol and further promote the apoptosis of HOS cells, we used an inhibitor of PERK (GSK2656157, $5 \mathrm{mM}$ ) to pretreat HOS cells for $1 \mathrm{~h}$ before celastrol treatment $(19,20)$. Following treatment with GSK2656157, the proliferation of HOS cells was further inhibited compared with that of the celastrol group; karyopyknosis, 
A

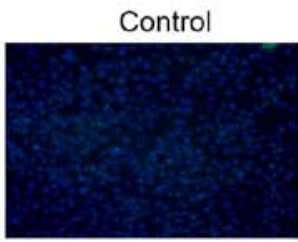

Celastrol

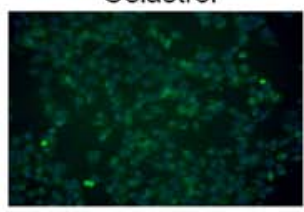

$\mathrm{C}_{10^{4}{ }^{42-Q 1}}$
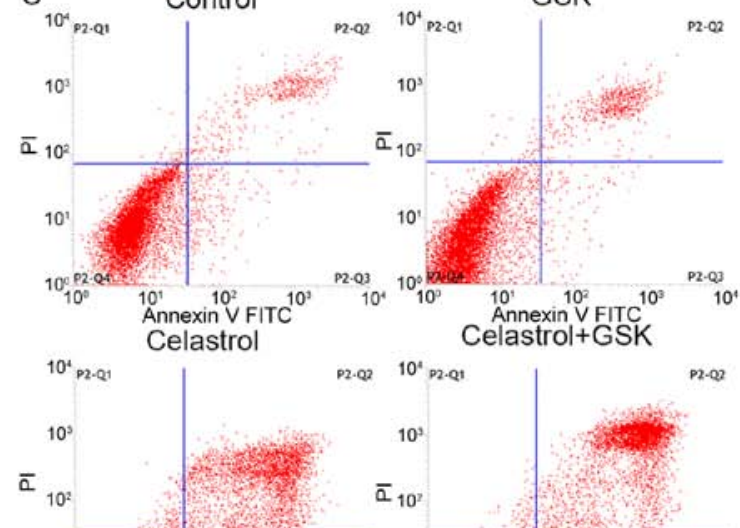

$10^{\prime}$
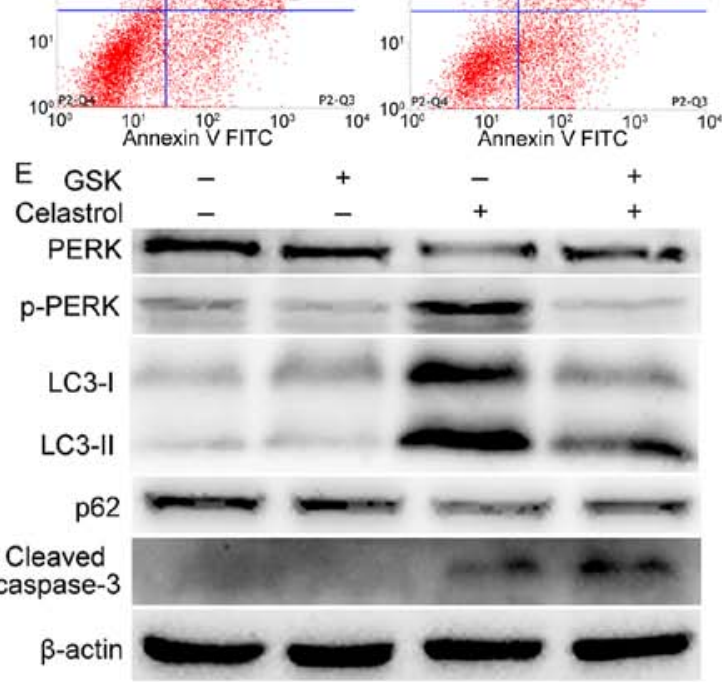

B

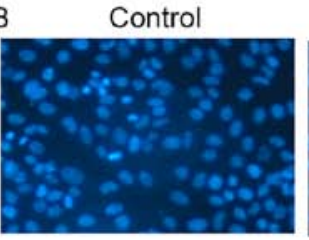

Celastrol+GSK
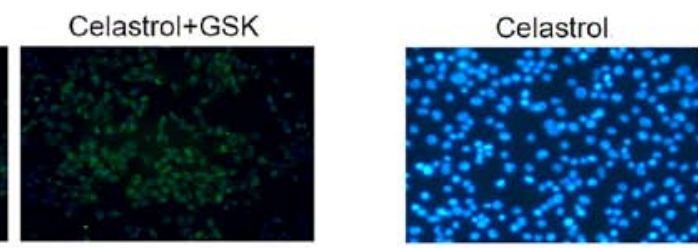

D

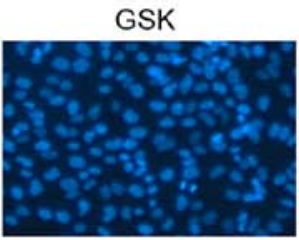

Celastrol+GSK
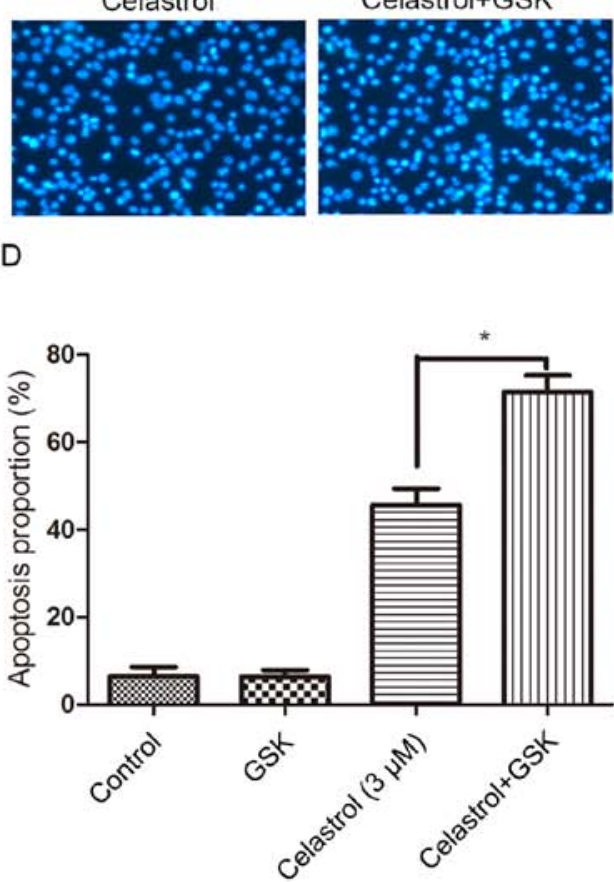

$F$ PERK 血 p62

$2.0 \times 0$ p-PERK $\square$ LC-3 I/LC-3 II

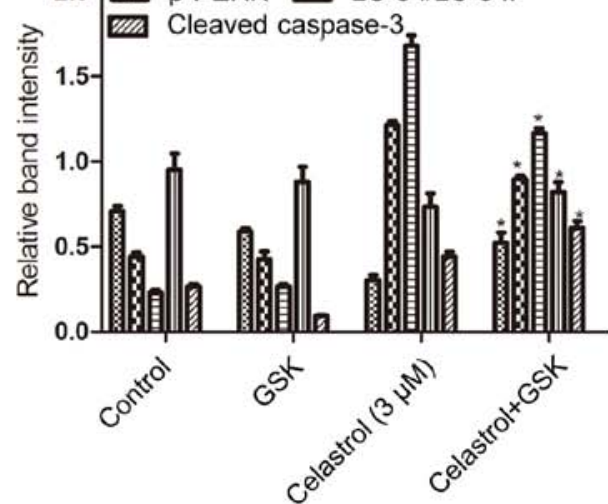

Figure 7. The PERK signaling pathway among ERS pathways has a protective effect on HOS cells. HOS cells were pretreated with GSK2656157 for $1 \mathrm{~h}$ in the presence or absence of celastrol. Some cells received no pretreatment or only GSK2656157. After the corresponding treatment, (A) the expression of PERK was determined by fluorescence microscopy (magnification, x200). (B) The apoptotic morphological changes of HOS cells were observed under fluorescence microscopy (magnification, $\mathrm{x} 200$ ). (C) The apoptotic rates were determined by FCM. ${ }^{*} \mathrm{P}<0.05$ vs. the celastrol group. (D) Cell viability was determined using the CCK-8 assay. (E and F) At $24 \mathrm{~h}$ following treatment, whole-cell lysates were prepared to assay PERK, p-PERK, Bip, LC-3, p62, and cleaved caspase-3 by western blotting. Data are presented as the mean \pm SD from three independent experiments. ${ }^{*} \mathrm{P}<0.05$ vs. the control group. ERS, endoplasmic reticulum stress; FCM, flow cytometry; CCK-8, Cell Counting Kit-8; SD, standard deviation.

condensation, karyorrhexis and other typical apoptotic bodies were more evident; and the apoptosis rates further increased, while immunofluorescence revealed that the expression of p-PERK was significantly attenuated (Fig. 7A-D; P<0.05). Following pretreatment with GSK2656157, the expression of PERK was inhibited, while cleaved caspase-3 was increased. All these results demonstrated that inhibiting the expression of PERK strongly promoted apoptosis induced by celastrol.
In addition, we found that pretreatment with GSK 2656157 attenuated autophagy and suppressed the expression of PERK (Fig. 7E-F; P<0.05).

\section{Discussion}

Despite the advances in medical technology for patients with osteosarcoma, long-term survival remains stagnant, and 
novel anti-osteosarcoma drugs are urgently needed. In addition, numerous studies have revealed that many components extracted from traditional Chinese medicine have antitumor effects $(3,8,10)$. Celastrol, extracted from the root of $T$. wilfordii, has been revealed to inhibit the proliferation of tumors, and the mitochondrial apoptotic pathway and death receptor apoptosis pathway were involved in the celastrol-induced apoptosis $(19,20)$. Research has reported the effect of celastrol on osteosarcoma, which has demonstrated great antitumor potential. Yu et al (21) found that celastrol negatively regulated cell invasion and migration ability of human osteosarcoma via the downregulation of the PI3K/Akt/NF- $\mathrm{KB}$ signaling pathway in vitro. However, there is still a lack of studies on the anticancer effect and the mechanism of celastrol on autophagy and apoptosis of osteosarcoma. Our results revealed that celastrol could inhibit cell viability, induce morphological changes of apoptosis and increase the apoptosis rate of HOS cells compared with control cells.

The functions of the ER, including protein synthesis, transmembrane transport, and modification following translation, play a key role in cell survival. The ER also serves as the largest intracellular calcium library and regulates intracellular calcium homeostasis. The concentration of calcium ions in the cytoplasm increases when the ER is damaged. Following treatment with celastrol, evident swelling and dysfunction of the ER appeared, with a significant increase in the cytoplasmic free calcium ion level, which indicated that the ER of HOS cells was damaged (22-25). The ER is also the receptor of cellular stress. When the ER is stimulated by damage factors, misfolded or folded proteins accumulate so that ERS is induced and the UPR is further promoted. When the UPR is induced, the heat shock protein Bip is normally located in the ER, the ERS transduction proteins IRE-1, ATF6 and PERK convert from a binding state into a dissociative state, and PERK becomes phosphorylated while the level of PERK decreases to activate downstream chaperone proteins and gene transcription related to protein secretion, which will improve the processing capacity of the ER for unfolded or misfolded proteins $(26,27)$. Thus, Bip is used as a marker protein for ERS. ERS will activate calnexin, Ero1-L $\alpha$, PDI and other chaperone proteins to promote the proper folding of unfolded or misfolded proteins, which alleviates the breakdown of cells. The results of this experiment revealed that the expression of Bip, calnexin, Erol-L $\alpha$, PDI, IRE1 $\alpha$ and p-PERK increased, while PERK decreased, indicating that ERS was induced by celastrol in HOS cells.

ERS is a protective mechanism for cells, but when prolonged excessive stress or dysfunction occurs, apoptosis may be induced (28-30). Apoptosis mediated through ERS is mainly induced by the PERK/EIF2 $\alpha$ signaling pathway activation of the ERS-specific transcription factors CHOP/GADD153. Apoptosis is mediated by the specific transcription factors CHOP/GADD153 of ERS through the PERK/EIF2 $\alpha$ signaling pathway or by the specific caspase-12 of ERS to activate apoptosis initiation factor caspase-9 and apoptosis executive factor caspase-3. Thus, elevated expression levels of $\mathrm{CHOP}$ and caspase-12 are markers of ERS-mediated apoptosis in ERS cells (31). The results of western blotting revealed that following treatment with celastrol, the expression of CHOP, cleaved caspase-12 and cleaved caspase-3 markedly increased, which indicated that ERS was involved in the apoptosis induced by celastrol. Furthermore, TG and DMSO were used as a positive control test to exclude false-positive results and the impact of DMSO. The mitochondrial pathway is an important mechanism in apoptosis for tumors. In addition, Bcl-2, Bax and cytochrome $c$ are regarded as the indexes of the mitochondrial pathway. The results of western blotting revealed that following treatment for 6,12 and $24 \mathrm{~h}$, the expression of Bax and cytochrome $c$ was increased and Bcl-2 was decreased, which indicated that the mitochondrial pathway was involved in the apoptosis of HOS cells induced by celastrol.

Moderate ERS facilitated intracellular environmental homeostasis and maintain cell survival, while long-lasting ERS injured cells. We clarified the specific role of ERS in HOS cells, and following pretreatment with TUDCA, FCM and western blotting revealed that apoptosis and autophagy induced by celastrol increased, while ERS was attenuated. In our previous research, we have found that external factors could induce cytoprotective autophagy against apoptosis or enhance pro-apoptotic and pro-autophagic effects on human osteosarcoma cells $(11,32)$. In the present study, the pro-apoptotic and pro-autophagic effects of celastrol on human osteosarcoma cells have already been confirmed by Li et al (10). Therefore, the results demonstrated that ERS inhibited autophagy and apoptosis and played a protective role in HOS cells. To suppress the protective function of ERS, further promote apoptosis and enhance the chemotherapeutic effect of celastrol, we sought to reverse the target of HOS-cell resistance to celastrol. Studies have found that PERK is the key factor of the PERK/EIF2 $\alpha$ signaling pathway $(33,34)$. Salaroglio et al $(13)$ reported that PERK molecules mediated colon cancer cell resistance to ERS and chemotherapeutic agents. Feng et al (35) found that the activation of PERK signaling molecules attenuated the ischemic injury of neuronal cells after melatonin preconditioning (35). Following pretreatment with GSK2656157, the results of CCK-8, fluorescence staining with Hoechst 33258, FCM and western blotting demonstrated that following inhibition of p-PERK expression, apoptosis induced by celastrol was further increased, which reversed the tolerance of HOS cells to celastrol. All the results indicated that the PERK/EIF $2 \alpha$ signaling pathway of ERS played a protective role in the apoptosis of HOS cells induced by celastrol. Inhibition of the expression of p-PERK attenuated the effect of ERS and further promoted apoptosis of HOS cells. Pretreatment with TUDCA inhibited the occurrence of ERS and the phosphorylation of PERK and promoted autophagy, while pretreatment with GSK2656157 inhibited the phosphorylation of PERK but attenuated autophagy. ERS, the PERK/EIF2 $\alpha$ signaling pathway and autophagy are usually triggered simultaneously due to the same stimulus, but their complicated relationships are controversial. Thus, the details and relationships among ERS, the PERK/EIF2 $\alpha$ signaling pathway and autophagy are still unclear and warrant further studies in more than one cell line and in vivo experiments.

In conclusion, the ERS pathway, the mitochondrial pathway and autophagy were involved in the apoptosis of HOS cells induced by celastrol. The ERS pathway not only protected HOS cells from apoptosis but also mediated apoptosis. Further research found that the expression of p-PERK increased, while ERS was induced, which contributed to the resistance to celastrol. These results will enrich our understanding of 
the mechanisms mediating celastrol-induced tumor cell death and the relationship among ERS, autophagy and apoptosis. Further studies are required to determine how to regulate the relationship of ERS, apoptosis and autophagy to enhance the anti-osteosarcoma effect of celastrol.

\section{Acknowledgments}

Not applicable.

\section{Funding}

The present study was supported by the National Natural Science Foundation of China (81572634).

\section{Availability of data and materials}

The datasets used during the present study are available from the corresponding author upon reasonable request.

\section{Authors' contributions}

YC carried out the experimental work and the data collection and interpretation. YO, YT and HL participated in the design and coordination of experimental work and acquisition of data. $\mathrm{HYi}$, $\mathrm{SZ}$ and $\mathrm{HYu}$ participated in the study design, data collection, analysis of data and preparation of the manuscript. $\mathrm{ZZ}$ and $\mathrm{BH}$ carried out the study design, the analysis and interpretation of data and drafted the manuscript. All authors read and approved the manuscript and agree to be accountable for all aspects of the research in ensuring that the accuracy or integrity of any part of the work are appropriately investigated and resolved.

\section{Ethics approval and consent to participate}

Not applicable.

\section{Patient consent for publication}

Not applicable.

\section{Competing interests}

The authors declare that they have no competing interests.

\section{References}

1. Ando K, Heymann MF, Stresing V, Mori K, Rédini F and Heymann D: Current therapeutic strategies and novel approaches in osteosarcoma. Cancers (Basel) 5: 591-616, 2013.

2. Arai K, Sakamoto R, Kubota D and Kondo T: Proteomic approach toward molecular backgrounds of drug resistance of osteosarcoma cells in spheroid culture system. Proteomics 13: 2351-2360, 2013.

3. Hu M, Luo Q, Alitongbieke G, Chong S, Xu C, Xie L, Chen X, Zhang D, Zhou Y, Wang Z, et al: Celastrol-induced Nur 77 interaction with TRAF2 alleviates inflammation by promoting mitochondrial ubiquitination and autophagy. Mol Cell 66: 141-153.e6, 2017.

4. Zhang R, Zhang N, Zhang H, Liu C, Dong X, Wang X, Zhu Y, $\mathrm{Xu}$ C, Liu L, Yang S, et al: Celastrol prevents cadmium-induced neuronal cell death by blocking reactive oxygen species-mediated mammalian target of rapamycin pathway. Br J Pharmacol 174: 82-100, 2017.
5. Li PP, He W, Yuan PF, Song SS, Lu JT and Wei W: Celastrol induces mitochondria-mediated apoptosis in hepatocellular carcinoma Bel-7402 cells. Am J Chin Med 43: 137-148, 2015.

6. Ni H, Zhao W, Kong X, Li H and Ouyang J: NF-kappa B modulation is involved in celastrol induced human multiple myeloma cell apoptosis. PLoS One 9: e95846, 2014.

7. Sethi G, Ahn KS, Pandey MK and Aggarwal BB: Celastrol, a novel triterpene, potentiates TNF-induced apoptosis and suppresses invasion of tumor cells by inhibiting NF-kappaB-regulated gene products and TAK1-mediated NF-kappaB activation. Blood 109: 2727-2735, 2007.

8. Li Z, Zhang J, Tang J and Wang R: Celastrol increases osteosarcoma cell lysis by $\gamma \delta \mathrm{T}$ cells through up-regulation of death receptors. Oncotarget 7: 84388-84397, 2016.

9. Yoon MJ, Lee AR, Jeong SA, Kim YS, Kim JY, Kwon YJ and Choi KS: Release of $\mathrm{Ca}^{2+}$ from the endoplasmic reticulum and its subsequent influx into mitochondria trigger celastrol-induced paraptosis in cancer cells. Oncotarget 5: 6816-6831, 2014.

10. Li HY, Zhang J, Sun LL, Li BH, Gao HL, Xie T, Zhang N and Ye ZM: Celastrol induces apoptosis and autophagy via the ROS/JNK signaling pathway in human osteosarcoma cells: An in vitro and in vivo study. Cell Death Dis 6: e1604, 2015.

11. Huang Q, Ou YS, Tao Y, Yin H and Tu PH: Apoptosis and autophagy induced by pyropheophorbide- $\alpha$ methyl estermediated photodynamic therapy in human osteosarcoma MG-63 cells. Apoptosis 21: 749-760, 2016.

12. Chiang CK, Wang CC, Lu TF, Huang KH, Sheu ML, Liu SH and Hung KY: Involvement of Endoplasmic Reticulum Stress, Autophagy, and Apoptosis in Advanced Glycation End Products-Induced Glomerular Mesangial Cell Injury. Sci Rep 6: 34167, 2016.

13. Salaroglio IC, Panada E, Moiso E, Buondonno I, Provero P, Rubinstein $\mathrm{M}$, Kopecka $\mathrm{J}$ and Riganti C: PERK induces resistance to cell death elicited by endoplasmic reticulum stress and chemotherapy. Mol Cancer 16: 91, 2017.

14. Zheng X, Xu F, Liang H, Cao H, Cai M, Xu W and Weng L: SIRT1/HSF1/HSP pathway is essential for exenatide-alleviated, lipid-induced hepatic endoplasmic reticulum stress. Hepatology 66: 809-824, 2017.

15. Kimura K, Mamane A, Sasaki T, Sato K, Takagi J, Niwayama R, Hufnagel L, Shimamoto Y, Joanny JF, Uchida S, et al: Endoplasmic-reticulum-mediated microtubule alignment governs cytoplasmic streaming. Nat Cell Biol 19: 399-406, 2017.

16. Qi L, Tsai B and Arvan P: New insights into the physiological role of endoplasmic reticulum-associated degradation. Trends Cell Biol 27: 430-440, 2017.

17. Rani S, Sreenivasaiah PK, Kim JO, Lee MY, Kang WS, Kim YS, Ahn Y, Park WJ, Cho C and Kim DH: Tauroursodeoxycholic acid (TUDCA) attenuates pressure overload-induced cardiac remodeling by reducing endoplasmic reticulum stress. PLoS One 12: e0176071, 2017.

18. Uppala JK, Gani AR and Ramaiah KVA: Chemical chaperone, TUDCA unlike PBA, mitigates protein aggregation efficiently and resists ER and non-ER stress induced HepG2 cell death. Sci Rep 7: 3831, 2017.

19. Zhang X, Yang J, Chen M, Li L, Huan F, Li A, Liu Y, Xia Y, Duan JA and Ma S: Metabolomics profiles delineate uridine deficiency contributes to mitochondria-mediated apoptosis induced by celastrol in human acute promyelocytic leukemia cells. Oncotarget 7: 46557-46572, 2016.

20. Kapoor S: Tumor growth attenuating effect of celastrol in systemic malignancies. Int J Cancer 139: 1431, 2016.

21. Yu X, Wang Q, Zhou X, Fu C, Cheng M, Guo R, Liu H, Zhang B and Dai M: Celastrol negatively regulates cell invasion and migration ability of human osteosarcoma via downregulation of the PI3K/Akt/NF- $\mathrm{KB}$ signaling pathway in vitro. Oncol Lett 12: 3423-3428, 2016.

22. Santofimia-Castano P, Izquierdo-Alvarez A, Plaza-Davila M, Martinez-Ruiz A, Fernandez-Bermejo M, Mateos-Rodriguez JM, Salido GM and Gonzalez A: Ebselen impairs cellular oxidative state and induces endoplasmic reticulum stress and activation of crucial mitogen-activated protein kinases in pancreatic tumour AR42J cells. J Cell Biochem 119: 1122-1133, 2018.

23. Takata T, Ihara H, Hatano N, Tsuchiya Y, Akaike T and Watanabe Y: Reactive sulfur species inactivate $\mathrm{Ca}^{2+} /$ calmodulin-dependent protein kinase IV via S-polysulfidation of its active-site cysteine residue. Biochem J 474: 2547-2562, 2017.

24. Bononi A, Giorgi C, Patergnani S, Larson D, Verbruggen K, Tanji M, Pellegrini L, Signorato V, Olivetto F, Pastorino S, et al: BAP1 regulates IP3R3-mediated $\mathrm{Ca}^{2+}$ flux to mitochondria suppressing cell transformation. Nature 546: 549-553, 2017. 
25. Scorrano L, Oakes SA, Opferman JT, Cheng EH, Sorcinelli MD, Pozzan T and Korsmeyer SJ: BAX and BAK regulation of endoplasmic reticulum $\mathrm{Ca}^{2+}$ : A control point for apoptosis. Science 300: 135-139, 2003.

26. Hetz C and Saxena S: ER stress and the unfolded protein response in neurodegeneration. Nat Rev Neurol 13: 477-491, 2017.

27. Badiola N, Penas C, Miñano-Molina A, Barneda-Zahonero B, Fadó R, Sánchez-Opazo G, Comella JX, Sabriá J, Zhu C, Blomgren $\mathrm{K}$, et al: Induction of ER stress in response to oxygenglucose deprivation of cortical cultures involves the activation of the PERK and IRE-1 pathways and of caspase-12. Cell Death Dis 2: e149, 2011.

28. Cerezo M, Lehraiki A, Millet A, Rouaud F, Plaisant M, Jaune E, Botton T, Ronco C, Abbe P, Amdouni H, et al: Compounds triggering ER stress exert anti-melanoma effects and overcome BRAF inhibitor resistance. Cancer Cell 29: 805-819, 2016.

29. López I, Tournillon AS, Prado Martins R, Karakostis K, Malbert-Colas L, Nylander K and Fåhraeus R: p53-mediated suppression of BiP triggers BIK-induced apoptosis during prolonged endoplasmic reticulum stress. Cell Death Differ 24: $1717-1729,2017$.

30. Guha P, Kaptan E, Gade P, Kalvakolanu DV and Ahmed H: Tunicamycin induced endoplasmic reticulum stress promotes apoptosis of prostate cancer cells by activating mTORC1. Oncotarget 8: 68191-68207, 2017.
31. Klionsky DJ, Abdelmohsen K, Abe A, Abedin MJ, Abeliovich $\mathrm{H}$, Acevedo Arozena A, Adachi H, Adams CM, Adams PD, Adeli K, et al: Guidelines for the use and interpretation of assays for monitoring autophagy (3rd edition). Autophagy 12: 1-222, 2016.

32. Tu P, Huang Q, Ou Y, Du X, Li K, Tao Y and Yin H: Aloe-emodin-mediated photodynamic therapy induces autophagy and apoptosis in human osteosarcoma cell line MG 63 through the ROS/JNK signaling pathway. Oncol Rep 35: 3209-3215, 2016.

33. Gupta A, Hossain MM, Miller N, Kerin M, Callagy G and Gupta S: NCOA3 coactivator is a transcriptional target of XBP1 and regulates PERK-eIF2 $\alpha$-ATF4 signalling in breast cancer. Oncogene 35: 5860-5871, 2016

34. Chiu TL and Su CC: Tanshinone IIA increases protein expression levels of PERK, ATF6, IRE1 $\alpha$, CHOP, caspase 3 and caspase 12 in pancreatic cancer BxPC 3 cell derived xenograft tumors. Mol Med Rep 15: 3259-3263, 2017.

35. Feng D, Wang B, Wang L, Abraham N, Tao K, Huang L, Shi W, Dong Y and Qu Y: Pre-ischemia melatonin treatment alleviated acute neuronal injury after ischemic stroke by inhibiting endoplasmic reticulum stress-dependent autophagy via PERK and IRE1 signalings. J Pineal Res 62: e12395, 2017. 